\title{
RUDJER BOŠKOVIĆ INSTITUTE RADIOCARBON MEASUREMENTS VII
}

\author{
DUŠAN SRDOC,, NADA HORVATINC̆IC, and BOGOMIL OBELIC \\ Rudjer Bošković Institute, POB 1016, 41001 Zagreb, Yugoslavia
}

and

\section{ADELA SLIEPGEVIC}

\author{
Faculty of Veterinary Medicine, University of Zagreb
}

\begin{abstract}
Results of extensive and systematic radiocarbon dating of tufa samples and associated materials (wood, plants, moss, and peat) are presented in this paper. Samples were collected in Plitvice Lakes National Park, Central Croatia, Yugoslavia, where intensive precipitation of tufa has taken place over several geologic epochs in a typical Karst area. Problems associated with radiocarbon dating of tufa are discussed in a previous paper (Srdoč et al, 1980). The authors concluded that dating of tufa is feasible and reliable, at least in the specific case of Karst regions, where retention of groundwater is short. Tufa is precipitated as a result of decomposition of groundwater bicarbonates due to loss of $\mathrm{CO}_{2}$, usually in waterfalls and cascades, and it is practically contemporaneous with living organic material. However, radiocarbon activity of modern tufa is lower than that of organic material due to dissolution of inactive carbonates (limestone) in groundwater. The initial activity of groundwater depends on the geology and vegetation of the area and it is widely discussed in the literature (Geyh, 1973). The initial activity, $A_{0}$, of tufa could be easily measured on recent samples, had not bomb-test contamination upset the equilibrium. Namely, while the atmospheric $\mathrm{CO}_{2}$ and living matter responded relatively quickly to the injection of radiocarbon in the atmosphere, groundwater and, consequently, tufa showed a much slower and damped response. Therefore, we have based our estimation of the initial activity of tufa on measurements of samples of known age collected before the bomb-test era. An average value of the initial activity equal to $85 \%$ of modern has been obtained which is in accordance with data for groundwater activity in similar areas. Consequently, all radiocarbon ages of tufa samples in this paper are based on the Libby half-life of ${ }^{14} \mathrm{C}$ (5568 years) and the initial radiocarbon activity equal to $85 \%$ of modern. The modern standard is 0.95 of the activity of NBS oxalic acid. Errors quoted are based on counting statistics only and do not include uncertainties in ${ }^{14} \mathrm{C}$ half-life and in initial activity. The latter may introduce much larger errors, up to \pm 1000 years, depending on the sampling site, as explained in the following sections. Whereas radiocarbon dating of Holocene tufa beds give reliable ages within the above specified errors, radiocarbon dating of old tufa beds is still questionable. Intrusion of minute quantities of modern calcareous material renders the ages of old $(\sim 30,000 \mathrm{yr})$ tufa useless. Research on measuring the age of old tufa deposits by other methods is in progress.
\end{abstract}


Preparation of wood, plant, and peat samples included pretreatment with boiling in $4 \% \mathrm{HCl}$ and $4 \% \mathrm{NaOH}$. Tufa samples were mechanically cleaned by scraping off surface layers containing algae, moss, and/or lichens. Tufa, which contains up to $97 \% \mathrm{CaCO}_{3}$, was dissolved in diluted $\mathrm{HCl}$ and evolved $\mathrm{CO}_{2}$ was frozen in a liquid $\mathrm{N}_{2}$ trap. Subsequent counting, gas preparation, and purification is described in $R, 1979, v 21, p$ 321-328, and the counting technique in R, 1971, v 13, p 135-140. Statistical processing of data has been computerized (Obelić and Planinić, 1977).

\section{ACKNOWLEDGMENTS}

This research was made possible by the cooperation and financial support of Plitvice Lakes National Park Administration. We thank J Movčan for continued help and encouragement. A Brnek-Kostić and M $\mathrm{Krga}$, whose close cooperation enabled us to collect samples and discuss many important aspects of natural phenomena in the Park area, deserve special thanks.

The following experts took part in various stages of research, field and laboratory work, discussions, and sample collection: $\mathrm{H}$ Moser, W Stichler, and $\mathrm{H}$ Batsche, all of Institut für Radiohydrometrie, GSF, Munich, West Germany, M Geyh, Niedersächsisches Landesamt für Bodenforschung, Hannover, West Germany, and S Božičević, Inst for Geology, Zagreb.

E Hernaus helped in sample preparation, V Andreić and P Hojski in electronics and maintenance, and $\mathrm{A}$ Turković in data processing.

GEOLOGIC, LIMNOLOGIC, AND BOTANICAL SAMPLES

\section{Surface sampling}

Most tufa samples were coll within Park area $\left(44^{\circ} 50^{\prime} \mathrm{N}, 15^{\circ} 35^{\prime} \mathrm{E}\right)$ on surface of barriers, outcrops, and dry river beds without digging or drilling, except for Gavanovac series where existing cutting through tufa barrier was used to coll samples from depth of tufa bed.

\section{Crna Rijeka series}

A strong karstic spring feeds Crna Rijeka R. Tufa is not precipitated at the spring, which has fairly constant temperature throughout the year $\left(8-10^{\circ} \mathrm{C}\right)$. Recent tufa is found ca $3 \mathrm{~km}$ downstream where brook forms cascades. Comment (DS): lowest radiocarbon activity of groundwater and recent tufa in area.

\section{Z-707. Teslin mlin waterfall $1 \quad 63.9 \pm 0.6 \%$ modern}

Recent tufa, surface layer deposited on moss, left bank of Crna Rijeka, 3km downstream from karst spring Crna Rijeka; coll Oct 1979. Comment (DS, NH): groundwater activity at spring C Rijeka: $59.7 \pm$ $0.6 \%$ modern.

\section{Z-752. Teslin mlin waterfall 2}

$62.6 \pm 0.6 \%$ modern

Same as Z-707, right bank of Crna Rijeka. 


\section{Z-753. Teslin mlin waterfall 3}

$64.0 \pm 0.6 \%$ modern

Same as Z-707; coll July 1980.

\section{Bijela Rijeka series}

Several small-capacity karst springs feed Bijela Rijeka R, which merges with Crna Rijeka $\mathrm{R}$ to form Matica $\mathrm{R}$. Comment (DS): ${ }^{14} \mathrm{C}$ activity of Bijela Rijeka $R$ and recent tufa are much higher than those of Crna Rijeka R, indicating different catchment area and/or hydrogeol of groundwater.

\section{Z-754. Watermill 1}

$85.3 \pm 0.8 \%$ modern

Tufa deposited on growing moss (Cratoneurum commutatum). Coll June 1980. Comment (DS, NH): Bijela Rijeka stream water activity: $75.7 \%$ modern; coll Nov 1979, same site.

\section{Z-755. Watermill 2}

$$
74.6 \pm 0.6 \% \text { modern }
$$

\section{$1050 \pm 80$}

Thick tufa deposits, presently ca $6 \mathrm{~m}$ above stream level. Sample from top layer ca $0.5 \mathrm{~m}$ below ground surface.

\section{Z-684. Bijela Rijeka, dry tufa barrier}

$$
\begin{array}{r}
1.2 \pm 0.3 \% \text { modern } \\
+2000 \\
36,000+1600
\end{array}
$$

Old tufa barrier $15 \mathrm{~m}$ above present stream level, buried in alluvial deposits. NE flank opened during road construction. Deposition of tufa on moss can be easily recognized in structure of tufa beds.

\section{Plitvički Ljeskovac series}

Karst area at confluence of Crna Rijeka and Bijela Rijeka. Numerous old tufa barriers emerging from alluvial deposits as well as peat bogs are characteristic of landscape.

\section{Z-809. Monument site 1}

$$
\begin{array}{r}
1.3 \pm 0.3 \% \text { modern } \\
+2200 \\
36,000 \quad 1800
\end{array}
$$

Old tufa barrier, partly recrystallized, 15 to $20 \mathrm{~m}$ above and ca $70 \mathrm{~m}$ from Matica R. Moss structure recognizable in non-crystalline parts of barrier. Crystallized structure covered by flowstone-like layers.

\section{Z-936. Monument site 2}

$$
\begin{array}{r}
1.4 \pm 0.3 \% \text { modern } \\
+5100 \\
34,500+3200
\end{array}
$$

Old tufa barrier, block no. 3.

\section{Z-811. Confluence site 1}

$$
\mathbf{7 . 5} \pm \mathbf{0 . 3 \%} \text { modern }
$$

Dripstone or flowstone-like lining of tubing in solid tufa block. Tubes or channels were formed by decay of wood trunks or branches, 
structure of which is well-preserved. Comment (DS): calcareous linings are definitely younger than tufa matrix (see Z-809, -812) which forms huge blocks perforated by channels, indicating groundwater flow through old barrier. While old tufa morphology and abundance reflects paleoclimatic conditions similar to present climate, calcareous linings are amorphous and thin, indicating much colder climate and sparse vegetation. Thus, age of calcareous linings could be linked to interstadial thaw producing run-off and groundwater flow labeled by ${ }^{14} \mathrm{C}$ from thin vegetation layer consisting of algae, mosses, and lichens.

\section{Z-685. Confluence site 2}

$7.4 \pm 0.3 \%$ modern $\mathbf{2 0 , 5 0 0} \pm \mathbf{5 0 0}$

Calcareous lining similar to Z-811, same tufa barrier but different micro-location.

\section{Z-812. Confluence site 3}

$2.5 \pm 0.3 \%$ modern $29,000 \pm 1300$

Old tufa barrier, buried in alluvial deposits including terra rossa and calcareous sand. Sample taken on surface of outcrop, 10 to $15 \mathrm{~cm}$ from holes lined with flowstone (Z-685, -811).

Z-918. Confluence site 4

$0.1 \pm 0.3 \%$ modern $>\mathbf{3 7 , 0 0 0}$

Old tufa barrier, block no. 2; sample taken 15 to $20 \mathrm{~cm}$ laterally of natural drain hole (Z-685).

\section{Z-919. Confluence site 5}

$$
\begin{array}{r}
0.9 \pm 0.3 \% \text { modern } \\
+6500 \\
37,000+3500
\end{array}
$$

Old tufa barrier, block no. 1 ; sample taken 10 to $15 \mathrm{~cm}$ above natural drain hole (Z-811).

\section{Z-813. Confluence site 6}

$$
0.0 \pm 0.3 \% \text { modern }
$$

Outcrop of tufa in marshy field, often flooded during high water; sample taken 20 to $25 \mathrm{~m}$ from old tufa barrier (Z-812).

\section{Z-920. Tufa quarry}

$$
\begin{array}{r}
1.5 \pm 0.3 \% \text { modern } \\
+1700 \\
33,500
\end{array}
$$

Sample taken from old tufa quarry where tufa was cut for building. Quarry is inoperative since foundation of National Park.

\section{Z-921. Vukmirović house}

$$
\begin{array}{r}
0.7 \pm 0.3 \% \text { modern } \\
>\mathbf{3 7 , 0 0 0}
\end{array}
$$

Tufa used for construction of walls, presumably from tufa quarry (Z-920). Comment (DS, NH): date confirms use of old tufa for building, although much younger Holocene tufa was found in other buildings in Park area. 
Z-700. Ex Bio-station

$$
\begin{array}{r}
56.5 \pm 0.5 \% \text { modern } \\
3350 \pm 100
\end{array}
$$

Outcrops of tufa in marshy field; moss structure evident. Present flood area. Comment (DS): sample belongs to widespread group of Holocene tufa barriers. Both Holocene and pre-glacial tufas are found in same area, showing influence of geomorphol of karstic terrain on location of barriers; climatic conditions governed timing of growth of tufa.

\section{Rječica series}

Rječica Creek has no karst springs. Many woodland brooks form Rječica Creek. No recent tufa formations were found in woodland brooks. Recent tufa precipitation starts at first waterfall, although old Holocene tufa was found in Rječica stream bed upstream from present waterfall.

\section{Z-748. Rječica waterfall 1}

86.4 $\pm 0.7 \%$ modern

Recent tufa, surface layer deposited on moss (Cratoneurum commutatum). Comment (DS, NH): Rječica stream water activity: $87.7 \%$ modern; sample coll June 1980, same site.

\section{Z-927. Rječica waterfall 2}

$78.7 \pm 0.6 \%$ modern $600 \pm 100$

Small tufa barrier formed around waterfall, now above stream level. Date confirms recent tufa formation.

\section{Z-750. Rječica waterfall 3}

$77.4 \pm 0.6 \%$ modern

Recent tufa in form of stump. Wood structure well-preserved.

$$
750 \pm 90
$$

\section{Z-925. Rječica 1}

$81.0 \pm 0.6 \%$ modern spring.

Thick tufa deposits, porous moss-like structure, Rječica tributary

\section{Z-924. Rječica 2}

$80.4 \pm 0.6 \%$ modern $430 \pm 110$

Thick tufa deposit, porous and amorphous structure, adjacent to Z-925. Comment (DS): tufa structure reflecting deposition patterns does not influence radiocarbon content significantly (see also Z-922, -923).

\section{Z-923. Rječica 3}

$80.2 \pm 0.6 \%$ modern

Inactive tufa barrier covered by humus and organic detritus. Typical porous moss-like structure (Cratoneurum commutatum).

Z-922. Rječica 4

$80.0 \pm 0.6 \%$ modern

Porous, amorphous tufa from same barrier as Z-923. 


\section{Z-746. Rječica 5}

$78.6 \pm 0.6 \%$ modern

Porous, amorphous tufa from same deposit as Z-924; different microlocation. Comment (DS, NH): consistent with previous observations, ${ }^{14} \mathrm{C}$ tends to spread fairly uniformly throughout thick tufa deposits during formative period. Thus, average age of whole deposit is obtained rather than detailed stratigraphy.

\section{Z-691. Rječica riverbed 1}

$66.0 \pm 0.6 \%$ modern $2050 \pm 100$

Tufa from dry stream bed. Waterfall on Rječica $R 1 \mathrm{~km}$ downstream from bridge.

\section{Z-926. Rječica riverbed 2}

$$
\mathbf{5 1 . 7} \pm \mathbf{0 . 5} \% \text { modern }
$$
$4080 \pm 110$

Tufa from stream bed, flooded at medium and high water.

\section{Z-747. Rječica riverbed 3}

$$
\begin{array}{r}
40.1 \pm 0.5 \% \text { modern } \\
6200 \pm 90
\end{array}
$$

Tufa from stream bed, flooded at high water. Comment (DS, NH): oldest Holocene sample. Repeated sampling at nearby site gave younger date (Z-926).

\section{Cave Garden series}

Cave Garden area covers $200 \mathrm{~m}$ consisting of tufa barrier full of caves, galleries, and pits. Tufa barrier became partly inactive in historic times due to human interference with environment.

\section{Z-663. Broken tufa stalactite}

$$
\text { 74.1 } \pm 0.3 \% \text { modern }
$$

Surface layer, flowstone structure.

\section{Z-664. Broken tufa stalactite 2}

$73.8 \pm 0.3 \%$ modern

Porous mossy structure, $25 \mathrm{~cm}$ below surface.

\section{Z-665. Broken tufa stalactite 3}

$$
74.8 \pm 0.4 \% \text { modern }
$$

Porous mossy structure, $53 \mathrm{~cm}$ below surface.

\section{Z-666. Broken tufa stalactite 4}

74.4 $\pm 0.4 \%$ modern $1070 \pm 100$

Porous mossy structure, $83 \mathrm{~cm}$ below surface. Comment (DS): data shows uniform distribution of ${ }^{14} \mathrm{C}$ in thick tufa column.

\section{Z-824. Broken tufa column 1}

$$
\begin{array}{r}
82.0 \pm 0.3 \% \text { modern } \\
270 \pm 100
\end{array}
$$

Base, surface layer, porous structure.

Z-911. Broken tufa column 2

$$
\begin{array}{r}
69.3 \pm 0.3 \% \text { modern } \\
1650 \pm 100
\end{array}
$$

Shaft, surface layer, flowstone structure. 


\section{Z-912. Broken tufa column 3}

Core at broken surface, $1.2 \mathrm{~m}$ from edge.

\section{Z-913. Broken tufa column 4}

Core at broken surface, $1 \mathrm{~m}$ from edge. Comment (DS): data support theory of "tubular growth" of tufa under favorable conditions. Uppermost and outer layers are older than core. Tufa is deposited progressively on inner wall of duct until it becomes choked.

\section{Z-821. Rock shelter 1}

$78.2 \pm 0.6 \%$ modern $660 \pm 90$

Sample from roof of rock shelter where tufa stalactite (Z-633 to -666) and column (Z-824 and -911 to -913) originated.

\section{Z-823. Rock shelter 2}

78.4 $\pm 0.6 \%$ modern $630 \pm 100$

Sample from bottom of rock shelter; thick sediment filled with clastic material.

\section{Z-909. Tufa cave 1}

$70.6 \pm 0.6 \%$ modern

Sample from bottom of small cave, $5 \mathrm{~m}$ deep. Well-preserved imprints of leaves (Fagus sylvatica) found in sediments.

\section{Z-910. Tufa cave 2}

$$
73.6 \pm 0.6 \% \text { modern }
$$$$
1160 \pm 100
$$

Tufa column dividing cave, surface layer.

\section{Z-953. Vertical wall 1}

$73.5 \pm 0.6 \%$ modern $1160 \pm 110$

Inactive tufa barrier, 12 to $15 \mathrm{~m}$ high, vertical surface layer, $1.5 \mathrm{~m}$ from bottom of barrier.

\section{Z-955. Vertical wall 2}

$82.8 \pm 0.7 \%$ modern

Fracture in tufa barrier, filled with dripstones.

\section{Z-954. Cave garden}

$77.0 \pm 0.6 \%$ modern $785 \pm 110$

Inactive tufa barrier in form of numerous caves, galleries, and channels. Sample from deepest cave. Comment (DS): radiocarbon activity of samples taken from bottom of tufa caves is not lower than that coll in open space, indicating simultaneous deposition of tufa throughout barrier during formative period of barrier growth.

\section{Plitvica Creek series}

Plitvica Creek forms waterfall, $86 \mathrm{~m}$ high, at confluence of Korana R. No tufa forms at karst spring Izvor Plitvice. Although short, Plitvica Creek is interesting for study of tufa deposition. Starting with cold water and no tufa precipitation at spring, stream water gradually warms, forming thin tufa coating on moss and branches $2 \mathrm{~km}$ downstream from spring. 
Forming several cascades with thick tufa deposits right before waterfall, $86 \mathrm{~m}$ high, Plitvica Creek ends in Korana $\mathrm{R}, 8$ to $10^{\circ} \mathrm{C}$ warmer. Loss of $\mathrm{CO}_{2}$ through aeration and decrease of $\mathrm{CaCO}_{3}$ solubility due to warming of stream water are responsible for intensive tufa formation in Plitvica Waterfall area.

\section{Z-805. Plitvica 1}

$86.8 \pm 0.7 \%$ modern

Recent tufa deposited on moss growing on reef. Comment (DS, NH): Plitvica stream water activity: $85.2 \%$ modern, coll Oct 1980.

\section{Z-701. Plitvica 2}

$91.2 \pm 0.7 \%$ modern

Recent tufa deposited on moss (Cratoneurum commutatum) $2 \mathrm{~km}$ downstream from Z-805 sampling site. Comment (DS, NH): higher activity agrees with observed downstream increase of radiocarbon content of stream and lake water (see Plitvice waters series).

\section{Z-952. Plitvica 3}

$41.7 \pm 0.5 \%$ modern

Dry bed of creek above Plitvica waterfall, $86 \mathrm{~m}$ high. $5860 \pm 130$

\section{Z-806. Plitvica 4}

$75.9 \pm 0.8 \%$ modern

Active tufa barrier at Hajduković Mill, surface layer, ca $10 \mathrm{~cm}$ thick.

\section{Z-706. Plitvica 5}

$65.4 \pm 0.6 \%$ modern

Thick deposit of tufa adjacent to Plitvica waterfall.

$$
2140 \pm 110
$$

\section{Z-831. Hajduković Mill 1}

$59.2 \pm 0.5 \%$ modern $2960 \pm 110$

Pit, 2 to 3m deep in flat terrace ("polje") near Hajduković Mill. Terrace is made entirely of tufa deposited by Plitvica $\mathbf{R}$. Pits are result of collapsing of cave roof. Sample taken $80 \mathrm{~cm}$ below ground surface.

\section{Z-802. Hajduković Mill 2}

$61.0 \pm 0.6 \%$ modern $2710 \pm 110$

Sample coll $80 \mathrm{~cm}$ below ground on opposite side of same pit. Date confirms fairly uniform activity within layer.

Z-803. Hajdukovié Mill 3

$55.6 \pm 0.6 \%$ modern $3480 \pm 110$

Sample from ditch in tufa connecting pit and Plitvica creek.

Z-801. Hajduković Mill 4

$45.0 \pm 0.5 \%$ modern $\mathbf{5 2 3 0} \pm \mathbf{1 3 0}$

Tufa from hollow wood trunk buried in thick tufa deposit, ca $2.5 \mathrm{~m}$ below ground, $0.5 \mathrm{~m}$ above bottom of pit.

Z-935. Hajduković Mill 5

$48.8 \pm 0.6 \%$ modern

Tufa surrounding hollow wood trunk in pit. $4550 \pm 140$ 
Z-800. Hajduković Mill 6

63.5 $\pm 0.6 \%$ modern $3730 \pm 110$

Wood from tree (conifer) buried in tufa, $2.5 \mathrm{~m}$ below ground in pit. Comment (DS): age of wood and adjacent tufa agree fairly well if initial activity of tufa is taken equal to 0.7 of modern standard, which is reasonable value for Karst area. Better agreement cannot be expected because tufa did not grow on surface of tree; it was transported and deposited by floods.

\section{Smolčića pećina cave series}

Outcrop of old tufa, ca $60 \mathrm{~m}$ above present level of Korana $\mathrm{R}$, on steep right flank of gorge. Tufa deposits mark extinct tributary of Korana R.

\section{Z-711. Smolěića pećina 1}

$0.5 \pm 0.3 \%$ modern

Tufa from bottom of horizontal cave, ca $12 \mathrm{~m}$ deep, ca $60 \mathrm{~m}$ above level of Korana R. Porous and amorphous structure.

Z-745. Smolčića pećina 2

$$
\begin{array}{r}
2.9 \pm 0.3 \% \text { modern } \\
28,000 \pm 900
\end{array}
$$

Tufa from bottom of cave, typical mossy structure.

Z-930. Smolčića pećina 3

$$
\begin{gathered}
1.5 \pm 0.3 \% \text { modern } \\
+5000 \\
\mathbf{3 3 , 0 0 0}
\end{gathered}
$$$$
-\mathbf{3 4 0 0}
$$

Same as Z-745, from another micro-location within cave.

\section{Z-742. Smolěića pećina 4}

Tufa from entrance to cave.

\section{Z-741. Smolčić plateau 1}

Outcrops of tufa emerging from soil in flat area right above cave roof. Tufa beds are probably inter-connected. Sample taken from surface of tufa block no. 1 .

Z-744. Smolěić plateau 2

Tufa, block no. 1, mossy structure, very hard.

Z-743. Smolčić plateau 3

Tufa, block no. 2, surface layer, very hard.

\section{Z-934. Smolěić plateau 4}

$$
\begin{array}{r}
2.2 \pm 0.3 \% \text { modern } \\
\mathbf{3 0 , 0 0 0} \pm 1700
\end{array}
$$

$$
8.0 \pm 0.3 \% \text { modern }
$$$$
19,600 \pm 300
$$

Tufa, block no. 1 , surface layer removed, sample taken ca 10 to $15 \mathrm{~cm}$ from surface. Due to tufa porosity, precipitation or surface water can easily penetrate thick tufa deposits. 


\section{Z-933. Smolčić plateau 5}

$$
\begin{array}{r}
1.0 \pm 0.3 \% \text { modern } \\
+8600 \\
36,500+4000
\end{array}
$$

Tufa, block no. 3, very hard porous structure.

\section{Lake Ciginovac series}

Tufa samples with various morphologies were coll in cave above Lake Ciginovac. Measurement should reveal any difference in radiocarbon content caused by depositional patterns or influence of external contamination on tufa in variety of morphologic forms and structures. Samples of tufa in form of powder (Z-814), fine-grained compact sediment (Z-815), compact thick coating on limestone rock (Z-816), petrified moss (Z-817), and dripstone (Z-818) were measured. Comment (DS, NH): in most cases, powdered or structureless tufa is older than mossy structured tufa. Dripstone or flowstone layers are always youngest, which can be explained by subsequent formation of calcareous coating on tufa surface.

Z-814. Ciginovac 1

$68.9 \pm 0.6 \%$ modern $1700 \pm 110$

Powdered tufa coll from crevice in tufa barrier, NE shore of Lake Ciginovac.

\section{Z-815. Ciginovac 2}

$69.7 \pm 0.6 \%$ modern $1610 \pm 110$

Fine-grained compact tufa, same site as Z-814.

\section{Z-816. Ciginovac 3}

$70.9 \pm 0.6 \%$ modern $1470 \pm 100$

Tufa layer, $3 \mathrm{~cm}$ thick, on bedrock, same site as Z-814.

\section{Z-817. Ciginovac 4}

72.4 $\pm 0.6 \%$ modern $1300 \pm 100$

Tufa in form of petrified moss (Cratoneurum commutatum); same site as Z-814.

\section{Z-818. Ciginovac 5}

$74.9 \pm 0.6 \%$ modern $1020 \pm 100$

Tufa covered by dripstone on surface. Outer layer containing mostly dripstone; same site as Z-814.

\section{Z-820. Ciginovac plateau 1}

$68.9 \pm 0.6 \%$ modern

Inactive tufa barrier above Lake Ciginovac, NE shore. $1700 \pm 100$

\section{Z-904. Ciginovac plateau 2}

$71.3 \pm 0.6 \%$ modern

Tufa sample, dripstone form, coll in crevice in tufa barrier.

Z-905. Ciginovac plateau 3

$72.1 \pm 0.6 \%$ modern

Tufa in form of petrified moss. $1330 \pm 100$ 


\section{Upper Lakes}

\section{Z-956. Okrugljak}

$$
77.3 \pm 0.6 \% \text { modern }
$$

$2100 \pm 100$

Active tufa barrier, right flank, presently dry. Hard mossy structure, covered with flowstone on surface.

\section{Z-937. Galovac}

$63.5 \pm 0.5 \%$ modern

Inactive tufa barrier near lake Galovac. Coll and subm by A Brnek, Natl Park Adm.

\section{Z-917. Milino Jezero}

$$
70.5 \pm 0.6 \% \text { modern }
$$

$1520 \pm 80$

Inactive tufa barrier $10 \mathrm{~m}$ above and $50 \mathrm{~m}$ from Milino Jezero pond.

\section{Z-959. Pevalek Plaque}

$56.5 \pm 0.6 \%$ modern

Zone of intensive tufa formation full of cascades, waterfalls, and streams. Sample coll from isolated tufa cliff, presently dry but surrounded by cascades and waterfalls. Comment (DS): date confirms that tufa formed over long period, presumably entire Holocene.

\section{Z-958. Mali Prštavci}

$79.1 \pm 0.5 \%$ modern

Active tufa barrier, left flank. Sample coll in crevice close to waterfalls.

\section{Z-957. Veliki Prštavci}

$75.5 \pm 0.6 \%$ modern $2300 \pm 110$

Active tufa barrier, right flank. Sample coll in dry cave. Hard mossy structure.

\section{Gradina series}

Relatively isolated hill with remains of prehistoric ramparts ("gradina"). Comment (DS): outcrops of old tufa on top of hill puzzled early observers. Tufa barrier is autochthonous and belongs to old, preglacial formation. Thick tufa deposits at foothill are much younger Holocene features.

\section{Z-667. Gradina, hilltop 1}

$$
\begin{gathered}
1.6 \pm 0.3 \% \text { modern } \\
+2000 \\
33,000
\end{gathered}
$$

Remains of old tufa barrier, presently on top of Gradina hill, ca $30 \mathrm{~m}$ above lake level.

\section{Z-671. Gradina, hilltop 2} $2.1 \pm 0.3 \%$ modern

Same as Z-667, different micro-location. 


\section{Z-825. Gradina, hilltop 3}

$$
\begin{gathered}
2.1 \pm 0.3 \% \text { modern } \\
+2000 \\
31,000
\end{gathered}
$$

Same as Z-667 except sample coll in small dry cave, protected from rain and snow.

Z-826. Gradina, hilltop 4

$$
\begin{array}{r}
0.4 \pm 0.3 \% \text { modern } \\
>37,000
\end{array}
$$

Tufa powder coll from crevice in cave (see Z-825).

\section{Z-827. Gradina, hilltop 5}

$$
\begin{array}{r}
1.4 \pm 0.3 \% \text { modern } \\
+2100 \\
34,000+1700
\end{array}
$$

Outcrop of old tufa protruding through soil on top of Gradina hill. Hard, reddish tufa, mossy structure evident, contains iron oxide.

\section{Z-900. Gradina, foothill 1}

$$
61.6 \pm 0.6 \% \text { modern }
$$

Thick deposits of tufa partly covered by soil and organic detritus. Compact but rather soft tufa.

\section{Z-901. Gradina, foothill 2}

$65.1 \pm 0.6 \%$ modern $2170 \pm 100$

Tufa deposits, 3 to $4 \mathrm{~m}$ thick, forming caves, shelters, and galleries. Mossy structure well-preserved.

\section{Z-902. Gradina, foothill 3}

$62.4 \pm 0.6 \%$ modern

Same location as Z-901. Compact tufa, grained structure.

\section{Z-903. Gradina, foothill 4}

$64.6 \pm 0.6 \%$ modern

Same location as Z-901. Tufa, typical mossy structure (Cratoneurum commutatum).

\section{Burget series}

Two old tufa barriers, presently separated by lake water and recent tufa deposits. Comment (DS): contemporaneity of barriers helps construct contour lines of area in past.

\section{Z-914. Burget 1}

$62.6 \pm 0.6 \%$ modern $2500 \pm 110$

Old tufa barrier, submerged in Lake Burget, except crest. Hard, porous tufa sample coll from top of barrier.

\section{Z-915. Burget 2}

$63.8 \pm 0.6 \%$ modern

Old tufa barrier, above lake level.

\section{Z-939. Burgetići}

$$
\begin{array}{r}
76.6 \pm 0.7 \% \text { modern } \\
830+110
\end{array}
$$

\footnotetext{
Active barrier above Lake Kozjak; soft porous tufa.
} 


\section{Z-674. Kozjak 1}

$60.1 \pm 0.7 \%$ modern

Old tufa barrier, SW shore of Lake Kozjak, 15 to $20 \mathrm{~m}$ above lake level. Original structure well-preserved. Sample coll from surface of tufa block.

\section{Z-829. Kozjak 2}

$63.2 \pm 0.5 \%$ modern

Same site as Z-674. Tufa sample coll in cave.

$2420 \pm 100$

\section{Gavanovac series}

Cutting through tufa barrier was made during pathway construction in Park, near Lake Gavanovac. Cutting, $4 \mathrm{~m}$ high and $1.6 \mathrm{~m}$ wide, was suitable to coll samples from depth of tufa deposits, which would normally need drilling rig. Tufa samples ca $\mathrm{ldm}^{3}$ in size were coll from vertical profile, starting at path level, in steps of $40 \mathrm{~cm}$ up to $2.8 \mathrm{~m}$ high. Uppermost sample is from crown of barrier at $4.05 \mathrm{~m}$. Two samples were coll $2 \mathrm{~m}$ laterally from axis of vertical profile to check uniformity of ${ }^{14} \mathrm{C}$ distribution in horizontal layer. Five samples were coll in horizontal profile at $1.6 \mathrm{~m}$ above path level. Starting with surface layer, $5 \mathrm{~cm}$ thick, sampling proceeded at $10 \mathrm{~cm}$ steps up to $45 \mathrm{~cm}$. Comment (DS): no distinct gradient was found in any direction, except in horizontal profile, where small but systematic decrease of ${ }^{14} \mathrm{C}$ activity was detected. This is consistent with measurements of tufa samples from other sites (see Cave Garden series, above). ${ }^{14} \mathrm{C}$ tends to spread uniformly in tufa deposits during build-up process, obliterating much stratigraphy. ${ }^{14} \mathrm{C}$ age gives average of all tufa deposits. In most cases, build-up period is much shorter than age of tufa beds, which diminishes inherent error in ${ }^{14} \mathrm{C}$ dating of tufa.

\section{Vertical profile}

$57.5 \pm 0.5 \%$ modern

Z-620. G-1

$3200 \pm 90$ $10 \mathrm{~cm}$.

Cutting through dry tufa barrier; height $(\mathrm{H})=0 \mathrm{~m}$, depth $(\mathrm{D})=$

Z-621. G-2

$58.9 \pm 0.6 \%$ modern

$\mathrm{H}=0.4 \mathrm{~m}, \mathrm{D}=10 \mathrm{~cm}$.

$3000 \pm 100$

Z-622. G-3

$58.2 \pm 0.6 \%$ modern

$\mathrm{H}=0.8 \mathrm{~m}, \mathrm{D}=10 \mathrm{~cm}$.

$3100 \pm 110$

Z-662. G-4

$59.1 \pm 0.6 \%$ modern $2980 \pm 110$ profile.

Lateral sampling; $\mathrm{H}=0.8 \mathrm{~m}, \mathrm{D}=20 \mathrm{~cm}, 2 \mathrm{~m}$ right from vertical

Z-623. G-5

$62.6 \pm 0.6 \%$ modern

Vertical profile; $\mathrm{H}=1.2 \mathrm{~m}, \mathrm{D}=10 \mathrm{~cm}$.

$\mathbf{2 5 0 0} \pm \mathbf{8 0}$ 
Z-661. G-6

$58.5 \pm 0.6 \%$ modern $3060 \pm 110$

Lateral sampling; $\mathrm{H}=1.2 \mathrm{~m}, \mathrm{D}=20 \mathrm{~cm}, 2 \mathrm{~m}$ left from vertical profile.

Z-624. G-7

$60.4 \pm 0.6 \%$ modern $2800 \pm 100$

Vertical profile; $\mathrm{H}=1.2 \mathrm{~m}, \mathrm{D}=20 \mathrm{~cm}$.

Z-628. G-8

$62.8 \pm 0.3 \%$ modern $2480 \pm 100$

$\mathrm{H}=2 \mathrm{~m}, \mathrm{D}=20 \mathrm{~cm}$.

Z-633. G-9

$63.0 \pm 0.3 \%$ modern $2450 \pm 110$

Vertical profile, wood-shaped tufa; $\mathrm{H}=2.3 \mathrm{~m}$; original structure of tree trunks and branches well-preserved.

Z-630. G-10

$60.6 \pm 0.6 \%$ modern

$\mathrm{H}=2.4 \mathrm{~m}, \mathrm{D}=20 \mathrm{~cm}$.

$2770 \pm 100$

Z-644. G-11

$62.1 \pm 0.6 \%$ modern $2570 \pm 110$

$\mathrm{H}=2.6 \mathrm{~m}, \mathrm{D}=20 \mathrm{~cm}$.

Z-631. G-12

$61.5 \pm 0.6 \%$ modern

$\mathrm{H}=2.8 \mathrm{~m}, \mathrm{D}=20 \mathrm{~cm}$. $2640 \pm 110$

Z-632. G-13

$61.6 \pm 0.6 \%$ modern

Vertical profile, top of barrier; $\mathrm{H}=4.05 \mathrm{~m}, \mathrm{D}=20 \mathrm{~cm}$.

\section{Horizontal profile}

Z-625. G-14

$62.0 \pm 0.6 \%$ modern $2580 \pm 110$

Horizontal profile; $\mathrm{H}=1.6 \mathrm{~m}$, surface layer, $5 \mathrm{~cm}$ thick.

Z-626. G-15

$61.0 \pm 0.6 \%$ modern

$\mathrm{H}=1.6 \mathrm{~m}, \mathrm{D}=10 \mathrm{~cm}$. $2720 \pm 100$

Z-660. G-16

$59.8 \pm 0.6 \%$ modern $2880 \pm 100$

$\mathrm{H}=1.6 \mathrm{~m}, \mathrm{D}=20 \mathrm{~cm}$

Z-681. G-17

$59.6 \pm 0.5 \%$ modern

$\mathrm{H}=1.6 \mathrm{~m}, \mathrm{D}=30 \mathrm{~cm}$.

$2910 \pm 110$

Z-682. G-18

$58.7 \pm 0.6 \%$ modern

$\mathrm{H}=1.6 \mathrm{~m}, \mathrm{D}=40 \mathrm{~cm}$

$\mathbf{3 0 3 0} \pm \mathbf{1 1 0}$ 


\section{Korana River series}

Last thick deposits of tufa, $\mathrm{N}$ boundary of Natl Park. Tufa precipitation rate diminishes as Korana $\mathrm{R}$ slows down. Comment (DS, NH): high ${ }^{14} \mathrm{C}$ activity of freshwater and recent tufa.

\section{Z-704. Korana 1}

$90.9 \pm 0.7 \%$ modern

Recent tufa deposited on moss covering river bed. Comment (DS, $\mathrm{NH}$ ): Korana $\mathrm{R}$ water activity: $93.1 \%$ modern.

\section{Z-686. Korana 2}

$73.0 \pm 0.6 \%$ modern $1230 \pm 100$

Cutting through thick deposit of tufa, 6 to $7 \mathrm{~m}$ above Korana $\mathbf{R}$. Fine-grained structure.

\section{Milka Trnina Waterfalls series}

Area of copious tufa deposition.

\section{Z-658. MT-1}

$91.3 \pm 0.8 \%$ modern

Recent tufa deposited on moss growing on cliffs. Moss (Cratoneurum commutatum) is continually splashed or sprayed by lake water.

\section{Z-659. MT-2}

$90.0 \pm 0.7 \%$ modern

Soft tufa layer, 3 to $4 \mathrm{~cm}$ thick, deposited under growing moss. Moss roots and organic detritus removed.

\section{Underwater sampling}

Bathymetric measurements revealed underwater tufa barriers in several lakes. Downstream growth of new tufa barriers caused flooding and inactivation of upstream barriers which are now several meters below present lake surface. Samples coll 1980 by Pragoaquanaut scuba divers, Prague, ČSSR, led by J Svetly.

\section{Lake Gradinsko Jezero series}

Sample of tufa coll from surface of submerged tufa barrier.

\section{Z-832. Lake Gradinsko Jezero 1}

$72.8 \pm 0.6 \%$ modern $1250 \pm 100$

Underwater tufa barrier; grained structure covered with algae; depth: $5.5 \mathrm{~m}$. Algae, roots, and mud removed.

\section{Z-833. Lake Gradinsko Jezero 2}

$70.3 \pm 0.6 \%$ modern

Same as Z-832 but different micro-location.

Z-834. Lake Gradinsko Jezero 3

Same as Z-832; depth: $3 \mathrm{~m}$.

\section{Lake Kozjak series}

Samples of tufa coll from surface of submerged tufa barrier. 
Z-843. Lake Kozjak 1

$72.6 \pm 0.6 \%$ modern $1270 \pm 100$

Underwater tufa barrier, spongy structure on surface. SW flank of barrier; depth: $5.5 \mathrm{~m}$.

\section{Z-842. Lake Kozjak 2}

71.4 $\pm 0.6 \%$ modern

Same as Z-843, NE flank of barrier.

$1420 \pm 110$

\section{Z-838. Lake Kozjak 3}

$72.4 \pm 0.6 \%$ modern $1290 \pm 100$

Central sec of underwater tufa barrier. Spongy or "cauliflower" structure, filled with lake sediment in pores. Mud and surface layer of algae removed; depth: $7 \mathrm{~m}$.

\section{Z-840. Lake Kozjak 4}

$75.3 \pm 0.6 \%$ modern

Same as Z-838; depth: $12 \mathrm{~m}$.

\section{Z-841. Lake Kozjak 5}

$75.3 \pm 0.6 \%$ modern

Same as Z-838; depth: $15 \mathrm{~m}$.

$980 \pm 100$

\section{Pre-bomb-test samples of wood, moss, and tufa}

\section{Pevalek collection series}

${ }^{14} \mathrm{C}$ activity of recent tufa has not followed sharp increase of ${ }^{14} \mathrm{C}$ activity in biosphere caused by nuclear weapon tests. Increased activity of groundwater, tufa, and aquatic plants due to increased activity of atmospheric $\mathrm{CO}_{2}$ in past decades can be obtained by comparison of activity of recent material with that from pre-bomb-test era, providing that age of latter is known. We found several such samples of wood encrusted with tufa and moss with tufa substratum from colln of late academician Ivo Pevalek. Comment (DS): measurements of samples coll in 1937 and comparison with recent data leads to two important conclusions: 1) activity of recent tufa is comparable to activity of "pre-war" tufa (ca 1937). Depending on sampling site, activity of recent tufa varies from $85 \%$ to $91 \%$ modern, whereas activity of "pre-war" tufa varies from $71 \%$ to $88 \%$ modern. Range of activities of recent tufa was measured on 10 samples; "pre-war" tufa was measured on 2 samples only, 2) use of average initial activity of $85 \%$ modern may introduce error up to $1000 \mathrm{yr}$. However, low activity equal to $70 \%$ modern is found in limited region close to karst springs; largest part of tufa deposits is in lake area where ${ }^{14} \mathrm{C}$ activity is fairly uniformly distributed ca $85 \%$ modern. Thus, estimated error is $\pm 500 \mathrm{yr}$ for most samples.

\section{Z-847. Wood coated with tufa}

$98.1 \pm 0.7 \%$ modern

Wooden branch (Fagus sylvatica), $4 \mathrm{~cm}$ diam, coated with tufa layer, 2 to $3 \mathrm{~cm}$ thick. Coll 1937 by I Pevalek and stored in dry cabinet. Subm by A Brnek, Natl Park Admin. Micro-location not known. Comment (DS): Suess effect is evident even though entire area is not industrialized. 
Prevailing westerly winds could account for depleted ${ }^{14} \mathrm{C}$ activity between two World Wars.

\section{Z-848. Tufa coating on wood}

$88.5 \pm 0.7 \%$ modern

Tufa coating, 2 to $3 \mathrm{~cm}$ thick, on wood surface (sample Z-847). Tufa was scraped off and dissolved in HCl. Comment (DS): ratio of practically contemporaneous pre-bomb-test wood and tufa ${ }^{14} \mathrm{C}$ activities: $\mathrm{A}_{\mathrm{o}}=0.9$. This value is higher than $A_{0}=0.85$, which is used in this paper as average value. Higher and lower values of $A_{o}$ were also found, depending on sampling site location (see Z-857 and -855).

\section{Z-853. Plant stalks}

$92.9 \pm 0.8 \%$ modern

Wcody stalks (unid. sp), plant stalks, moss, and tufa from sample stored in cabinet. Coll by I Pevalek, ca 1937. Comment (DS): low ${ }^{14} \mathrm{C}$ content of relatively recent plants indicates absorption of $\mathrm{CO}_{2}$ depleted in ${ }^{14} \mathrm{C}$ from decomposition of hydrocarbonates.

\section{Z-855. Tufa substratum}

$68.8 \pm 0.6 \%$ modern

Surface layer, $3 \mathrm{~cm}$ thick, soft crumbly structure with moss roots. Roots and other organic material removed.

\section{Z-856. Tufa substratum \\ $69.6 \pm 0.8 \%$ modern}

Lower layer, $3 \mathrm{~cm}$ below surface, $3 \mathrm{~cm}$ thick, soft crumbly structure.

\section{Z-857. Moss}

$72.8 \pm 0.7 \%$ modern

Cratoneurum commutatum, grown on tufa, coll ca 1937 by I Pevalek; stored in dry condition in cabinet together with tufa substratum. Tufa removed mechanically and measured separately (see Z-855 and -856). Moss treated with $\mathrm{HCl}$ to remove remaining carbonates.

\section{Z-908. Wooden post}

$96.5 \pm 0.7 \%$ modern

Wooden post covered with tufa layer, 2 to $3 \mathrm{~cm}$ thick. Comment (DS): sample contains mostly sapwood and bark. Post was used to support small bridge on Labudovac plateau and was replaced. No record of this exists but ${ }^{14} \mathrm{C}$ activity of sapwood clearly show that wood was cut before bomb-test contamination.

\section{Z-907. Tufa coating on wooden post $81.6 \pm 0.7 \%$ modern}

Tufa coating, 2 to $3 \mathrm{~cm}$ thick, on wooden post (Z-908). Comment (DS): ratio between tufa and adjacent wood activities: $\mathrm{A}_{0}=0.845$.

\section{Botanical samples, Plitvice Park area}

Z-668. Terrestrial plant 1 $134.5 \pm 1.1 \%$ modern Dry leaves (Fagus sylvatica) coll Nov 1976, Lake Kozjak area.

\section{Z-656. Terrestrial plant 2}

$130.4 \pm 1.0 \%$ modern

Twigs (Salix cinerea) coll March 1978, M Trnina Waterfalls. Plant roots submerged in lake water. Comment (DS): lake water activity: $85 \%$ modern; atmospheric $\mathrm{CO}_{2}$ activity: $131.5 \%$ modern (March 1978). 
Z-657. Moss 1

$91.7 \pm 1.0 \%$ modern

Moss (Cratoneurum commutatum) coll March 1978, M Trnina Waterfalls. Moss growing on tufa substratum, constantly splashed with water.

Z-679. Moss 2

$82.4 \pm 0.7 \%$ modern

Moss (Cratoneurum commutatum) growing on tufa substratum, constantly submerged in lake water, Galovac cascades.

Z-678. Moss 3

$83.0 \pm 0.7 \%$ modern

Moss (Cratoneurum commutatum) growing on tufa substratum, presently dry, Galovac cascades area. Comment (DS): although exposed to atmospheric $\mathrm{CO}_{2}$, activity of which was $131.5 \%$, moss activity is much closer to lake water or recent tufa activity.

Z-677. Aquatic plant

77.4 $\pm 1.0 \%$ modern Galovac.

Aquatic plant (Potamogetum perfoliatus) coll Spring 1978, Lake

\section{Z-846. Submerged wood 1}

$89.5 \pm 0.7 \%$ modern position; depth: $9 \mathrm{~m}$, height: $7.5 \mathrm{~m}$. Sample taken from core of trunk.

\section{Z-845. Submerged wood 2}

$91.5 \pm 0.8 \%$ modern

Same as Z-846, outer layer of tree trunk. Comment (DS): rate of growth of tufa barrier crest can be calculated on assumption that tree was killed by flooding. Based on age of outer layer and depth, average growth rate of $1.3 \mathrm{~cm} / \mathrm{yr}$ is obtained. This agrees well with observations on recent rise of lake level.

\section{Surface water activity}

\section{Z-692. Crna Rijeka spring}

$59.7 \pm 0.6 \%$ modern

Karst spring, main water resource of area. Temperature fairly constant, averaging $7.8^{\circ} \mathrm{C}$. Tritium activity close to mean yearly activity of precipitation. Sample coll Oct 1979.

Z-694. Bijela Rijeka spring

$75.7 \pm 0.7 \%$ modern

Several karst springs feed Bijela Rijeka R. Sample coll Oct 1979 ca $300 \mathrm{~m}$ downstream from spring. Comment (DS, NH): abundant vegetation along stream increases ${ }^{14} \mathrm{C}$ activity which is probably lower at spring.

Z-942. Prošće

$78.7 \pm 0.7 \%$ modern Lake water, W shore of Prošćansko Jezero, coll Oct 1981.

Z-708. Plitvica $85.3 \pm 0.7 \%$ modern Stream water, tapped from karst spring, coll Oct 1979.

Z-710. Rječica

$88.8 \pm 0.7 \%$ modern

Stream water, coll Oct 1979. Rječica brook has no karst spring. 


\section{Z-672. Lake Kozjak 1}

$84.5 \pm 0.7 \%$ modern

Lake water from NW shore, coll April 1979.

Z-693. Lake Kozjak 2

89.3 \pm 0.7\% modern

Lake water co!l at outlet Oct 1979. Temperature of lake water is 8 to $10^{\circ} \mathrm{C}$ above karst spring temperature in summer.

Z-709. Korana River

$92.1 \pm 0.8 \%$ modern

River water coll Oct 1979 under bridge on Karlovac-Plitvička Jezera hwy. Intensive tufa precipitation ends at this point.

6. Peat samples

\section{Plitvički Ljeskovac peat bog series}

Peat bog near Plitvički Ljeskovac, Plitvice Lakes Natl Park. Luličina Bara peat bog is in area of intensive tufa precipitation. Peat samples were extracted with Dachnowsky-type hand-corer by A Šercelj for pollen analysis (Culiberg and Šercelj, 1981).

Samples were treated with $4 \% \mathrm{HCl}$ and $4 \% \mathrm{NaOH}$ at $80^{\circ} \mathrm{C}$, thoroughly washed and dried. Depth is given in $\mathrm{cm}$ below bog surface. Comment (DS): coincident beginning of tufa and peat formation in Holocene at ca $6200 \pm 200 \mathrm{yr}$ BP may be explained by possibility that tufa formation and intensive vegetation started simultaneously when climatic conditions at this alt $(640 \mathrm{~m})$ became favorable for both processes.

Z-636. 5 -30cm

$$
190 \pm 100
$$

Z-637. $30-60 \mathrm{~cm}$

$$
1050 \pm 70
$$

Z-640. $60-90 \mathrm{~cm}$

$$
1240 \pm 90
$$

Z-641. $90-120 \mathrm{~cm}$

$$
1670 \pm 70
$$

Z-642. $120-150 \mathrm{~cm}$

$$
1860 \pm 90
$$

Z-643. $150-180 \mathrm{~cm}$

$$
2060 \pm 100
$$

Z-638. $180-210 \mathrm{~cm}$

$$
2380 \pm 80
$$

Z-946. $300-320 \mathrm{~cm}$

$2830 \pm 110$

Z-947. $400-420 \mathrm{~cm}$

$3030 \pm 110$

Z-969. 440-460cm

$3140 \pm 110$

Z-960. $460-480 \mathrm{~cm}$

$3520 \pm 110$

Z-970. $540-560 \mathrm{~cm}$

$3810 \pm 110$

Z-949. 600-620cm

$3950 \pm 110$

Z-950. $680-700 \mathrm{~cm}$

$4580 \pm 120$

Z-972. 740-760cm

$5160 \pm 130$

Z-961. 840-860cm

$5530 \pm 130$ 
REFERENCES

Culiberg, Metka and Šcrcelj, A, 1981, Pollen analyses of the sediments of Plitvička Jezera (Lakes of Plitvice): Acta Bot Croatica, v 40, p 147-154.

Geyh, M A, 1973, On the determination of the initial ${ }^{14} \mathrm{C}$ content in groundwater, in Rafter, $T$ A and Grant-Taylor, eds, Internatl conf on radiocarbon dating, 8th, Proc: Wellington, New Zealand, Royal Soc New Zealand, p D58-D69.

Obelić, Bogomil and Planinić, J, 1977, Computer processing of ${ }^{14} \mathbf{C}$ and ${ }^{3} \mathbf{H}$ data; statistical tests and correction of data, in Internatl conf on low radioactivity measurements, Proc: The High Tatras, Slovenské pedagogické nakladatelstvo, Bratislava, p 117-120.

Srdoč, Dušan, Breyer, B, and Sliepčević, Adela, 1971, Rudjer Bošković Institute radiocarbon measurements I: Radiocarbon, v 13, p 135-140.

Srdoč, Dušan, Obelić, Bogomil, Horvatinčić, Nada, and Sliepčević, Adela, 1979, Measurement of the ${ }^{14} \mathrm{C}$ activity of the ANU Sucrose Secondary Standard by means of the proportional counter technique: Radiocarbon, v 21, p 321-328.

1980, Radiocarbon dating of calcareous tufa; how reliable data can we expect?, in Stuiver, Minze and Kra, Renee, eds, Internatl radiocarbon conf, 10th, Proc: Radiocarbon, v 22, no. 3, p 858-862. 